\title{
New IUPAC/CITAC guide available: Selection and use of proficiency testing schemes for a limited number of participants
}

The new guide jointly sponsored by IUPAC Analytical Chemistry Division, IUPAC Interdivisional Working Party on Harmonization of Quality Assurance and the Cooperation on International Traceability in Analytical Chemistry (CITAC) concerns implementation of proficiency testing (PT) schemes for a limited number of participating laboratories. Here 'limited number' refers to fewer than 30 . Therefore, the guide can be helpful for quality control of measurement and/or test results in small countries (with a limited number of laboratories acting in the same field), industries under development or in research, for environmental analyses, laboratory accreditation tasks, activities of a regulator, etc.

The metrological approach to the PT schemes is based on use of certified reference materials (CRMs) as test items with traceable property values, while the composition of these items is unknown to the participants.
If PT results shall qualify in the framework of the concept 'tested once, accepted everywhere', their metrological traceability, comparability and compatibility is mandatory. The approach described in this Guide grants also the possibility of assessing the performance of groups of PT participants or of the entire collective by comparing the PT consensus values with the certified values of the test items. The Guide features tabulated criteria for this assessment and practical examples are described for illustration of the issues discussed.

The Guide has been published in Pure Appl. Chem. (PAC) online on 18 February 2010 as an ASAP article. It is available from http://www.iupac.org/publications/pac/asap/ PAC-REP-09-08-15/. Its printed version is scheduled to appear in the May issue of PAC.

(Source: www.citac.cc). 